\title{
DETERMINAÇÃO DE OLANZAPINA EM FORMULAÇÕES FARMACÊUTICAS POR ESPECTROFOTOMETRIA: DESENVOLVIMENTO E VALIDAÇÃO
}

\author{
Jardes Figuerêdo do Rêgo, Jacira Izidório de Moura e Graziella Ciaramella Moita* \\ Departamento de Química, Universidade Federal do Piauí, 64049-550 Teresina - PI, Brasil
}

Recebido em 7/3/09; aceito em 7/7/09; publicado na web em 8/1/10

\begin{abstract}
SPECTROPHOTOMETRIC OLANZAPINE DETERMINATION IN PHARMACEUTICAL FORMULATIONS: METHOD DEVELOPMENT AND VALIDATION. Spectrophotometric methods of zero order, first and second derived order had been developed for olanzapine determination in tablets using ethanol and isopropanol as solvent. The two solvents revealed to be adequate. For the three methods the calibration curve coefficient of correlation had been greater than 0.9998 with limit of detection varying from 0.068 to $0.190 \mathrm{mg} \mathrm{L}^{-1}$, in ethanol, and 0.026 to $0.205 \mathrm{mg} \mathrm{L}^{-1}$, in isopropanol. The inter-day precision was inferior to 1.1 and $1.9 \mathrm{mg} \mathrm{L}^{-1}$ for ethanol and isopropanol, respectively. The average recoveries varied from 98 to $101 \%$, in ethanol and 99 to $103 \%$ in isopropanol.
\end{abstract}

Keywords: olanzapine; UV spectrophotometry; validation.

\section{INTRODUÇÃO}

A olanzapina, 2-metil-4-(4-metil-1-piperazinil)-10H-tieno(2,3-b) $(1,5)$ benzodiazepina (Figura 1), é um agente psicotrópico pertencente à classe das tienobenzodiazepinas. ${ }^{1}$ Foi obtida pela primeira vez em 1982, na Inglaterra, por um grupo de pesquisadores da Eli Lilly and Company a partir de modificações estruturais da clozapina. Em 1986 começou a despertar o interesse da indústria farmacêutica como alternativa hematologicamente mais segura ao tratamento com clozapina e, em meados da década de 90, a olanzapina foi licenciada para comercialização no mercado europeu e norte-americano. ${ }^{2,3}$ No Brasil, somente em 1999 foi incluída na lista de medicamentos excepcionais do Ministério da Saúde e, desde então, se tornou uma das drogas mais utilizadas no tratamento dos sintomas positivos e negativos da esquizofrenia e demais transtornos relacionados., ${ }^{4,5}$

Essa substância faz parte do grupo de drogas denominadas de atípicas ou de segunda geração, cuja principal característica é promover a ação antipsicótica em doses nas quais os efeitos extrapiramidais não se manifestem de forma significativa, ${ }^{3}$ porém, provoca efeitos adversos, tais como ganho de peso (10\% ou mais), sonolência e/ou sedação. ${ }^{5,6}$

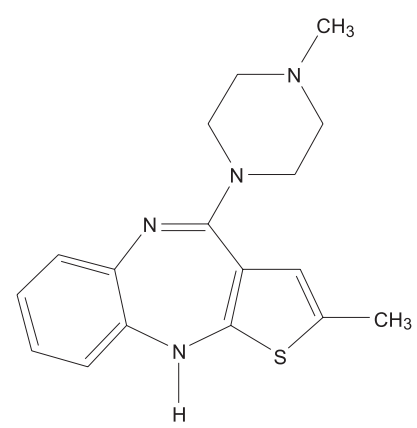

Figura 1. Estrutura da molécula de olanzapina

A olanzapina não é oficialmente registrada em nenhuma farmacopéia, ${ }^{7}$ por isso torna-se necessário o desenvolvimento e a validação de um método analítico que seja capaz de quantificar o fármaco nas especialidades farmacêuticas comercializadas no mercado. Existem na

*e-mail: graziella@ufpi.edu.br literatura científica vários estudos visando a quantificação de olanzapina em amostras biológicas e formulações farmacêuticas, dentre os quais se destacam os métodos cromatográficos,${ }^{8,9}$ eletroforéticos, ${ }^{10}$ eletroquími$\cos ,{ }^{10}$ espectrofotométricos, ${ }^{10-12}$ injeção em fluxo, ${ }^{1}$ cinéticos ${ }^{13}$ e titulométricos. ${ }^{12}$ No entanto, a maioria destes métodos apresenta inconvenientes como, por exemplo, uso de equipamentos caros, ${ }^{1,9,10}$ reagentes tóxicos ${ }^{10,12}$ e procedimentos demasiadamente complexos ${ }^{1,11}$ e demorados que os tornam pouco acessíveis e, em alguns casos, pouco viáveis.

Segundo as normas da Agência Nacional de Vigilância Sanitária ${ }^{14}$ (ANVISA), a validação de um método deve assegurar, através de estudos experimentais, que este atenda às exigências das aplicações analíticas, assegurando a confiabilidade dos resultados. Para tanto, deve apresentar especificidade, linearidade, intervalo de trabalho, precisão, sensibilidade, limite de quantificação e exatidão adequadas à análise.

O objetivo deste trabalho foi propor um método que possa quantificar a olanzapina em formulações farmacêuticas através da espectrofotometria molecular no ultravioleta, aliada a métodos derivativos, comparando-o com os já existentes na literatura. Os métodos propostos foram validados de acordo com o guia da ANVISA da resolução RE no 899 de 29 de maio de 2003.

\section{PARTE EXPERIMENTAL}

\author{
Materiais \\ Medicamentos \\ Foram estudadas as amostras de olanzapina na forma de compri- \\ mido, Zyprexa $^{\circledR}$ (Eli Lilly and Company, Lote: A498769, validade: \\ 03/2011) e uma produzida em uma planta piloto pelo Núcleo de \\ Tecnologia Farmacêutica da Universidade Federal do Piauí (NTF/ \\ UFPI), ambas a $10 \mathrm{mg}$.

\section{Reagentes} \\ Para calibração do método foi utilizada olanzapina de grau far- \\ macêutico (100,7 \%, Gamma $\left.{ }^{\circledR}\right)$, Lote: 050201, validade: 02/2008). \\ Os estudos foram realizados utilizando metanol 99,8\% (Vetec), \\ etanol 99,5\% (Synth e Nuclear), isopropanol 99,5\% (Synth, Vetec e \\ Reagen), propanol 99,0\% (Vetec), butanol 99,0\% (Reagen) e álcool \\ butílico terciário 99,0\% (Dinâmica). Para o estudo do $\mathrm{pH}$ foram uti- \\ lizados ácido clorídrico (Vetec) e hidróxido de sódio (Vetec). Todos \\ os reagentes utilizados foram de grau P.A.
}


Para o estudo dos interferentes, foram empregados os excipientes dióxido de silício coloidal (Pharma ${ }^{\circledR}$ ), croscarmelose sódica (Mingai $^{\circledR}$ ), celulose microcristalina 102 (Mingai ${ }^{\circledR}$ ), estearato de magnésio $\left(\right.$ Facispa $\left.^{\circledR}\right)$ e lactose anidra, os quais foram utilizados para a produção dos comprimidos na planta piloto do NTF.

\section{Equipamentos}

As medidas espectrofotométricas foram realizadas no espectrofotômetro Hitachi U-3000. Para o estudo de precisão interlaboratorial foi usado o espectrofotômetro Techcomp UV8500. Para as medidas de $\mathrm{pH}$ foi utilizado $\mathrm{pHmetro} \mathrm{Tecnal} \mathrm{Tec-3MP.} \mathrm{Para} \mathrm{a} \mathrm{centrifugação} \mathrm{das}$ amostras foi empregada a centrífuga Sigma 6-15. As massas foram medidas em uma balança analítica digital Denver Instrument APX 200.

\section{Programas computacionais}

Para a construção dos gráficos e tratamento estatístico dos dados foram utilizados os programas Origin 7.5 (OriginLab) e SPSS 15.0 for Windows (SPSS Inc.). Para derivação dos espectros foi utilizado o programa UV00 do espectrofotômetro Hitachi U-3000.

\section{Metodologia}

\section{Robustez}

A robustez do método foi avaliada através dos seguintes parâmetros: estabilidade das soluções, efeito do $\mathrm{pH}$ sobre o espectro de absorção e variação na marca do solvente.

Para o estudo de estabilidade foram preparadas soluções estoque de olanzapina a $100 \mathrm{mg} \mathrm{L}^{-1}$ em etanol ou isopropanol. Cada solução estoque foi distribuída em três frascos de mesmo volume, sendo um deles transparente e os demais âmbares. A solução armazenada no frasco transparente foi mantida sobre a bancada do laboratório exposta à luz ambiente (natural e artificial), durante o dia, e as demais foram envolvidas em papel alumínio. Destas duas soluções protegidas da luz, uma foi mantida à temperatura ambiente média de $25,0 \pm 5,0^{\circ} \mathrm{C}$ e a outra refrigerada a uma temperatura média de $4,0 \pm 3,0^{\circ} \mathrm{C}$. As soluções foram diluídas a $10 \mathrm{mg} \mathrm{L}^{-1}$ e os espectros foram obtidos diariamente durante um período de 10 dias e, posteriormente, a cada 5 dias, até completar 30 dias. Os resultados obtidos foram avaliados pela análise de variância ${ }^{15}$ (ANOVA, $\mathrm{p}=0,05)$ no comprimento de onda de máxima absorção $\left(\lambda_{\max }\right)$ da banda estudada e de mais 20 pontos experimentais localizados ao redor do $\lambda_{\max }$.

$\mathrm{O}$ efeito do $\mathrm{pH}$ sobre a absorbância foi realizado usando soluções preparadas com os dois solventes. Inicialmente prepararam-se soluções a $250 \mathrm{mg} \mathrm{L}^{-1}$ de olanzapina, sendo posteriormente diluídas a $10 \mathrm{mg} \mathrm{L}^{-1}$. Em seguida, o pH da solução foi ajustado com soluções de $\mathrm{HCl}$ a $0,01 \mathrm{~mol} \mathrm{~L}^{-1}$ ou $\mathrm{NaOH}$ a $0,01 \mathrm{~mol} \mathrm{~L}^{-1}$ para obtenção do $\mathrm{pH}$ ácido e básico, respectivamente. Os resultados obtidos foram avaliados pela análise de variância ${ }^{15}$ (ANOVA, $\mathrm{p}=0,05$ ) no comprimento de onda de máxima absorção da banda estudada e de mais 20 pontos experimentais localizados ao redor do $\lambda_{\max }$

O efeito da mudança de marca foi realizado com soluções a $10 \mathrm{mg}$ $\mathrm{L}^{-1}$, em cada solvente, obtidos de diferentes fabricantes. Os resultados obtidos foram avaliados pela análise de variância ${ }^{15}($ ANOVA, $p=0,05$ ) no comprimento de onda de máxima absorção da banda estudada e de mais 20 pontos experimentais localizados ao redor do $\lambda_{\max }$.

\section{Seletividade}

A seletividade do método foi realizada avaliando os espectros dos excipientes utilizados na produção dos comprimidos de olanzapina do NTF, em etanol e isopropanol, nas seguintes concentrações: lactose anidra $\left(312,0 \mathrm{mg} \mathrm{L}^{-1}\right)$, croscarmelose sódica $\left(3,75 \mathrm{mg} \mathrm{L}^{-1}\right)$, estearato de magnésio $\left(2,5 \mathrm{mg} \mathrm{L}^{-1}\right)$, dióxido de silício coloidal (2,5 $\left.\mathrm{mg} \mathrm{L}^{-1}\right)$ e celulose microcristalina $102\left(231,5 \mathrm{mg} \mathrm{L}^{-1}\right)$. O perfil dos espectros foi avaliado e comparado com o espectro de uma solução de olanzapina a $10,0 \mathrm{mg} \mathrm{L}^{-1}$.

\section{Linearidade}

Os espectros foram obtidos empregando-se os seguintes parâmetros: intervalo de varredura 190 a $400 \mathrm{~nm}$, velocidade de varredura $300 \mathrm{~nm} \mathrm{~min}{ }^{-1}$, a leitura foi realizada a cada $0,5 \mathrm{~nm}$, abertura de fenda 2,0 nm, lâmpada de deutério, linha de base system, cubeta de quartzo com caminho óptico de $1 \mathrm{~cm}$ e solvente (etanol ou isopropanol) como branco. Prepararam-se soluções com concentrações no intervalo de 5,0 a 50,0 $\mathrm{mg} \mathrm{L}^{-1}$. Os espectros de ordem zero (OZ) para cada uma das soluções foram suavizados em 15 pontos e, posteriormente, derivados utilizando-se o programa UV00 para obtenção dos espectros de $1^{\mathrm{a}}(1 \mathrm{D}), 2^{\mathrm{a}}(2 \mathrm{D}), 3^{\mathrm{a}}$ (3D) e $4^{\mathrm{a}}(4 \mathrm{D})$ ordem de derivada, com $\Delta \lambda$ variando de 1 a $10 \mathrm{~nm}$.

Para o espectro de ordem zero, foi determinada a absorbância em $\lambda_{\max }$. Para os espectros derivados, foram obtidos os valores das derivadas nos comprimentos de onda correspondentes ao máximo ou mínimo (dependendo da ordem da derivada) da banda. Para cada comprimento de onda selecionado, foi construída uma curva calibração, correlacionando o sinal (absorbância ou derivada) em função da concentração. As equações das retas foram obtidas através do método dos mínimos quadrados.

\section{Preparo das soluções da amostra}

Inicialmente os comprimidos a serem triturados foram pesados para obtenção da massa média que possibilita o cálculo da quantidade de amostra adequada para as análises.

Para o preparo da amostra Zyprexa ${ }^{\circledR}$, pesaram-se de 5 a 7 comprimidos e colocou-se em um tubo de ensaio, o qual foi imerso em nitrogênio líquido por um período de 20 a 30 min; em seguida, os comprimidos foram triturados em almofariz e homogeneizados. Pesou-se 0,2121 g do sólido pulverizado e preparou-se $50 \mathrm{~mL}$ de solução estoque em etanol ou isopropanol, com concentração final de $100 \mathrm{mg} \mathrm{L}^{-1}$ de olanzapina. Como as partículas dos excipientes em suspensão eram muito pequenas e não precipitaram, houve necessidade de centrifugação por um período de $30 \mathrm{~min}$ a $2.500 \mathrm{rpm}$. O sobrenadante foi separado e mantido em frasco âmbar à temperatura ambiente.

Para a amostra do NTF, pesou-se de 5 a 7 comprimidos, triturouse e homogeneizou-se. Pesou-se 0,1235 g do sólido pulverizado e preparou-se $50 \mathrm{~mL}$ de solução estoque em etanol ou isopropanol, com concentração de $200 \mathrm{mg} \mathrm{L}^{-1}$ de olanzapina. Como as partículas suspensas dos excipientes, para essa amostra, eram maiores que no caso do Zyprexa ${ }^{\circledR}$, elas precipitaram naturalmente após repouso por 30 min, protegidas da luz e à temperatura ambiente, não havendo necessidade de centrifugação. O sobrenadante foi separado e mantido em frasco âmbar à temperatura ambiente.

\section{Limites de detecção e quantificação}

Os limites de detecção e quantificação ${ }^{16}$ foram calculados, respectivamente, pelas expressões: $\mathrm{LOD}=3,3 \mathrm{~s} / \mathrm{b}$ e LOD $=10 \mathrm{~s} / \mathrm{b}$, onde $s$ é a estimativa do desvio padrão do sinal do branco e $b$ é a inclinação ou coeficiente angular da curva de calibração. Para obtenção destes limites foram obtidos 21 espectros do branco.

\section{Precisão}

Os ensaios de precisão ${ }^{16,17}$ foram realizados com amostras do comprimido Zyprexa ${ }^{\circledR}$. A precisão intradia foi avaliada em três níveis de concentração e em triplicata através do valor do desvio padrão relativo (DPR). As soluções estoque foram preparadas conforme especificado no tópico Preparo das soluções da amostra. Para o estudo foram 
utilizadas concentrações de 5,$0 ; 10,0 ; 15,0 \mathrm{mg} \mathrm{L}^{-1}$ de olanzapina.

A precisão interdia foi realizada após 2 dias, por um segundo analista, no mesmo laboratório, utilizando o mesmo espectrofotômetro (Hitachi).

Os ensaios interlaboratoriais foram desenvolvidos no laboratório da Farmácia Escola da UFPI, por um terceiro analista, utilizando outro espectrofotômetro (Techcomp). Para todos os experimentos adotouse o mesmo procedimento experimental e a concordância entre os resultados foi avaliada através do teste $F$ e teste $t$ pareado $(\mathrm{p}=0,05)$. Todos os experimentos foram realizados em triplicata.

\section{Exatidão}

A exatidão ${ }^{16,17}$ em etanol e isopropanol foi avaliada segundo o teste de adição e recuperação de padrão, em três níveis de concentração. Para o comprimido Zyprexa ${ }^{\circledR}$, transferiu-se quantitativamente $0,1061 \mathrm{~g}$ da amostra pulverizada para três balões volumétricos de 25 $\mathrm{mL}$. Adicionou-se 2,5; 5,0 e 7,5 mL da solução padrão estoque de concentração $500 \mathrm{mg} \mathrm{L}^{-1}$. Após o volume completado, a solução foi centrifugada por um período de $30 \mathrm{~min}$ a $2.500 \mathrm{rpm}$. Alíquotas de 1,0 $\mathrm{mL}$ de cada uma das três soluções foram transferidas para balões de $10 \mathrm{~mL}$ e os volumes completados com o solvente, obtendo-se concentrações finais de 5,0;10,0;15,0 $\mathrm{mg} \mathrm{L}^{-1}$ de padrão e de $10,0 \mathrm{mg} \mathrm{L}^{-1}$ de olanzapina da amostra. Para os comprimidos do NTF, preparou-se uma solução a $200 \mathrm{mg} \mathrm{L}^{-1} \mathrm{e}$, após decantação dos excipientes, retirouse $0,5 \mathrm{~mL}$ da solução sobrenadante e transferiu-se para três balões de $10 \mathrm{~mL}$. Em seguida, adicionou-se 0,5; 1,0 e 1,5 mL de solução padrão a $100 \mathrm{mg} \mathrm{L}^{-1}$, obtendo concentrações finais de 5,0;10,0;15,0 $\mathrm{mg} \mathrm{L}^{-1}$ de padrão e 10,0 $\mathrm{mg} \mathrm{L}^{-1}$ de olanzapina da amostra. Estes processos foram realizados em triplicata e a exatidão do método foi dada em termos de porcentagem de recuperação.

\section{RESULTADOS E DISCUSSÃO}

A olanzapina é insolúvel em água, mas solúvel em meio ácido e em solventes, como álcoois. ${ }^{11,17} \mathrm{Na}$ literatura ${ }^{9,10,12}$ são descritos métodos que utilizam metanol como solvente para análise espectrofotométrica de olanzapina na região do ultravioleta. Dos solventes testados a olanzapina apresentou boa solubilidade em metanol, etanol, propanol, isopropanol, butanol e álcool butílico terciário, sendo que alguns espectros foram bastante semelhantes. Desses solventes, optou-se por trabalhar apenas com etanol e isopropanol, descartando-se metanol pela sua toxicidade, butanol e álcool butílico terciário, devido a sua viscosidade, e proponal por apresentar espectro de absorção da olanzapina semelhante ao obtido para solução etanólica. Observa-se que o espectro obtido para olanzapina em etanol (Figura 2) apresenta três bandas bem definidas $(272,5 ; 226,5$ e $208,5 \mathrm{~nm})$ e em isopropanol (Figura 3) duas bandas (273,0 e 226,5 nm). Estas são resultado de transições do tipo $\pi \rightarrow \pi^{*},{ }^{19}$ características de compostos orgânicos que possuam ligações duplas conjugadas, e podem ser usadas para análise quantitativa.

Das três bandas do espectro da olanzapina em etanol (Figura 2), apenas duas são adequadas para análise quantitativa. A banda a 208,5 nm sofre deslocamento batocrômico com o aumento da concentração, o que resulta em desvio da Lei de Beer. Outro fator que torna inviável o uso dessa banda é que o etanol absorve ${ }^{19}$ abaixo de $210 \mathrm{~nm}$, causando interferência na análise.

Derivou-se os espectros de ordem zero, pois a espectrofotometria derivativa, que tem sido utilizada na análise de fármacos, ${ }^{20,21}$ pode eliminar sinais de substâncias interferentes, como excipientes, aumentando a seletividade do método. Os espectros da 3D e 4D apresentaram muito ruído, mesmos após a suavização, o que resulta em desvio elevado nas medidas e, portanto, são inadequados para análise quantitativa. Os espectros da 1D e 2D (Figuras 2 e 3 ) apresentaram pouco ruído após a suavização e houve aumento do sinal com relação ao espectro de ordem zero. Observou-se que, com o aumento de $\Delta \lambda$ no cálculo da derivada, ocorre uma diminuição dos detalhes do espectro e as bandas se tornaram mais intensas e com menos ruído, portanto, todas as análises foram realizadas utilizando $\Delta \lambda=10$. Em etanol, a derivada de primeira ordem apresenta apenas uma banda a $296,5 \mathrm{~nm}$ e a de segunda ordem possui duas bandas, em 310,0 e 275,0 $\mathrm{nm}$ (Figura 2). Em isopropanol foram utilizados os sinais em 297,0 $\mathrm{nm}$, no espectro de primeira ordem, e em 310,0 e $275,5 \mathrm{~nm}$, para o espectro de segunda ordem de derivada (Figura 3).

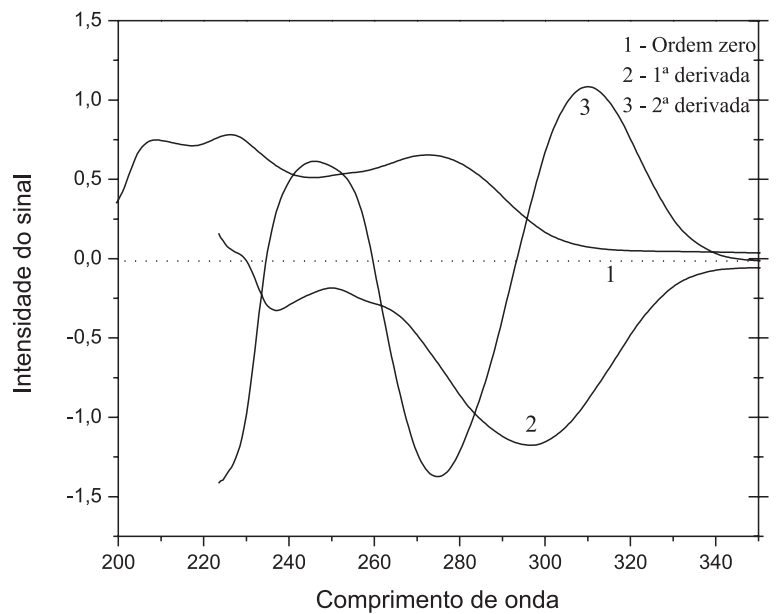

Figura 2. Espectro no ultravioleta de olanzapina em etanol (1); primeira derivada do espectro (2) e segunda derivada do espectro (3)

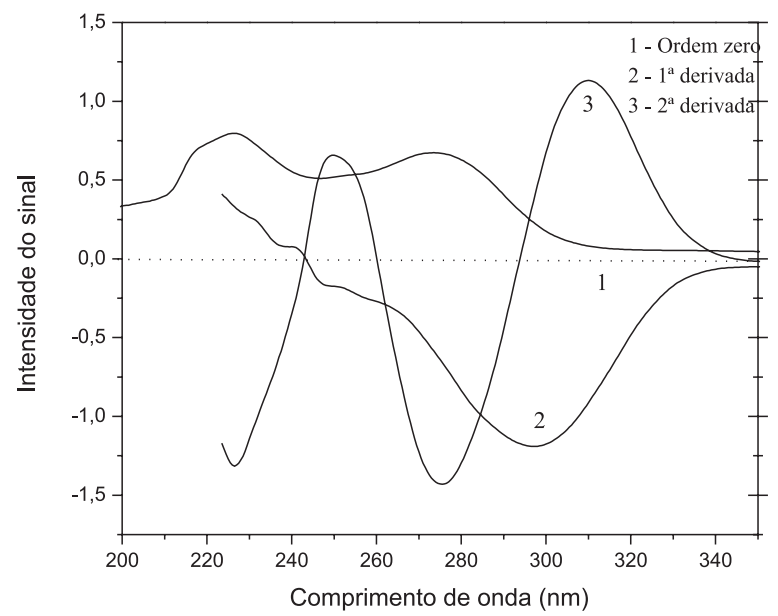

Figura 3. Espectro no ultravioleta de olanzapina em isopropanol (1); primeira derivada do espectro (2) e segunda derivada do espectro (3)

\section{Robustez}

\section{Estabilidade}

O estudo da estabilidade da olanzapina, em ambos os solventes, a partir do espectro de ordem zero, mostrou que até o $30^{\circ}$ dia as soluções, protegidas da luz, à temperatura ambiente ou sob refrigeração, permanecem com absorbâncias estatisticamente iguais, de acordo com análise de variância ${ }^{20}$ (ANOVA, $\mathrm{p}=0,05$ ). No entanto, as amostras que permaneceram expostas à luz apresentaram diminuição progressiva do sinal a partir do $4^{\circ}$ dia, em etanol, para a banda em $272,5 \mathrm{~nm}$ e do $10^{\circ}$ dia, em isopropanol, na banda em 273,0 nm (Figura 4). 


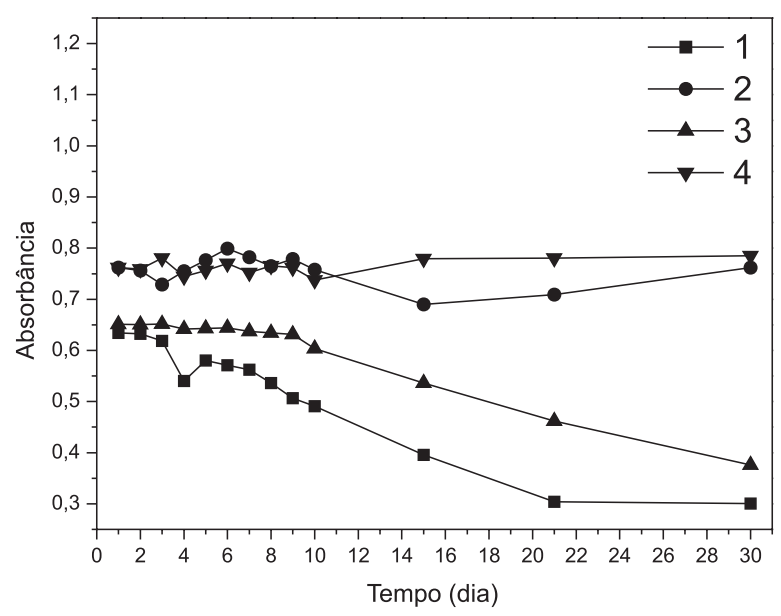

Figura 4. Absorbâncias das soluções de olanzapina na presença de luz: em etanol 272,5 nm (1); em etanol 226,5 nm (2); em isopropanol 273,0 nm (3) e em isopropanol $226,5 \mathrm{~nm}$ (4)

Foi observado que durante o período de análise a cor da solução que era amarela tornou-se mais intensa, indicando uma fotodecomposição da olanzapina. Isso mostra que uma solução de olanzapina apresenta sensibilidade luz, porém, quando protegida da mesma, pode ser estocada em temperaturas médias que variam de $4,0 \pm 3,0{ }^{\circ} \mathrm{C}$ (temperatura da geladeira) a $25,0 \pm 5,0{ }^{\circ} \mathrm{C}$ (temperatura ambiente).

\section{Efeito do pH sobre o espectro de absorção}

A Figura 5 mostra que o comportamento do sinal analítico é bastante similar para os dois solventes. Observa-se, em todos os casos, que em $\mathrm{pH}$ acima de 8,0 não há mudança significativa (ANOVA, $\mathrm{p}=0,05$ ) na absorbância nos comprimentos de onda estudados, o que indica que o método possui uma boa tolerância ao aumento do $\mathrm{pH}$.

Embora em $\mathrm{pH}$ acima de 8,0 haja estabilidade no sinal do espectro de olanzapina para as bandas estudadas, em ambos os solventes, optouse por não se realizar o ajuste do $\mathrm{pH}$, pois com adição de solução aquosa de $\mathrm{NaOH}$ há diminuição na solubilidade da olanzapina. $\mathrm{O}$ pH da solução de olanzapina a $10 \mathrm{mg}^{-1}$ é 7,33, em etanol, e 7,66, em isopropanol, bastante próximo daquele da região em que o sinal é estável. Em pH ácido foi observado que o sinal varia em ambos os solventes, devido a uma possível reação ácido base da olanzapina com $\mathrm{HCl}$.

\section{Efeito da mudança de marca do solvente}

Utilizando diferentes marcas de solventes, não houve alteração significativa (ANOVA, $\mathrm{p}=0,05$ ) na banda a $272,5 \mathrm{~nm}$, em etanol, e 273,0 nm, em isopropanol. Na banda em $226,5 \mathrm{~nm}$, em ambos os solventes, observou-se que, com a mudança de marca, houve uma pequena variação na intensidade do sinal analítico que pode ser atribuída à diferença na pureza dos solventes de diferentes marcas.

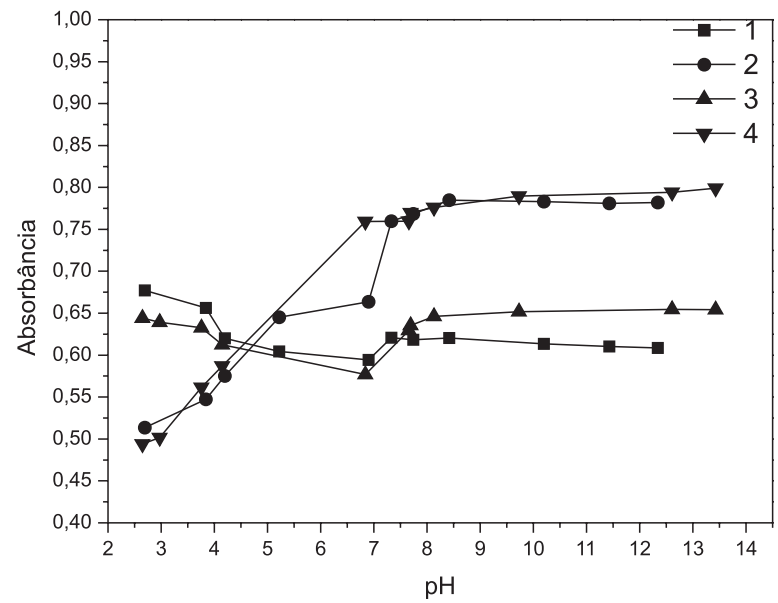

Figura 5. Absorbâncias das soluções de olanzapinaem diferentes pH: em etanol 272,5 nm (1); em etanol 226,5 nm (2); em isopropanol 273,0 nm (3) e em isopropanol $226,5 \mathrm{~nm}(4)$

\section{Seletividade}

Os estudos com os excipientes mostraram que nenhum deles apresenta solubilidade apreciável nos solventes utilizados; foi observado que a maioria precipitou, exceto o estearato de magnésio que permaneceu suspenso causando leve turbidez. A análise visual dos espectros das soluções dos excipientes, nos solventes estudados, mostra que nenhum deles absorve nos comprimentos de onda nos quais a olanzapina apresenta banda, portanto, não causam interferência nestes sinais. Em concentrações mais elevadas foi observado que a suspensão de estearato de magnésio se torna mais turva levando ao aumento do sinal analítico em toda a região espectral, devido ao espalhamento da radiação, podendo afetar a exatidão e precisão dos métodos. Esta interferência foi removida através da separação dos excipientes por centrifugação e decantação.

\section{Linearidade, limites de detecção e de quantificação}

As Tabelas 1 e 2 apresentam os parâmetros das curvas de calibração obtidas usando etanol e isopropanol como solvente. Através das equações das retas, observa-se que, tanto em etanol como em isopropanol, houve aumento da inclinação da curva de calibração e, consequentemente, na sensibilidade dos métodos derivados. Para todas as curvas de calibração, os coeficientes de correlação foram maiores que 0,9998 , os quais são melhores que o obtido por Jasiñska e Nalewajko ${ }^{1}(r=0,9983)$ que utilizaram reações de oxidação da olanzapina para obter um produto de coloração rosa-violeta. Os métodos desenvolvidos apresentam linearidade melhor que as estabelecidas pela $\operatorname{ANVISA}^{14}(r>0,99)$.

No estudo em etanol, o método $\mathrm{OZ}$ em $272,5 \mathrm{~nm}$ apresentou os menores limites de detecção e de quantificação, 0,068 e 0,208 mg L-1, respectivamente, e no estudo em isopropanol os menores limites de

Tabela 1. Parâmetros de calibração para os métodos analíticos propostos para quantificar olanzapina utilizando etanol como solvente

\begin{tabular}{lcccccc}
\hline Método & $\lambda(\mathrm{nm})$ & Equação & $\mathrm{r}$ & $\mathrm{LOD}\left(\mathrm{mg} \mathrm{L}^{-1}\right)$ & $\mathrm{LOQ}\left(\mathrm{mg} \mathrm{L}^{-1}\right)$ & $\mathrm{LOL}(\mathrm{mg} \mathrm{L}-1)$ \\
\hline $\mathrm{OZ}$ & 272,5 & $\mathrm{~A}=0,003+0,064 \mathrm{C}$ & 0,9999 & 0,068 & 0,208 & 50,0 \\
$\mathrm{OZ}$ & 226,5 & $\mathrm{~A}=0,018+0,076 \mathrm{C}$ & 0,9999 & 0,190 & 0,576 & 45,0 \\
1D & 296,5 & $\mathrm{D} 1=-0,004-0,116 \mathrm{C}$ & $-0,9999$ & 0,085 & 0,259 & 50,0 \\
2D & 310,0 & $\mathrm{D} 2=0,004+0,108 \mathrm{C}$ & 0,9999 & 0,159 & 0,482 & 50,0 \\
2D & 275,0 & $\mathrm{D} 2=-0,007-0,136 \mathrm{C}$ & $-0,9998$ & 0,168 & 0,510 & 50,0 \\
\hline
\end{tabular}

OZ - Ordem zero, 1D - Primeira ordem e 2D Segunda ordem. LOD - Limite de detecção, LOQ - Limite de quantificação e LOL - Limite de linearidade 
Tabela 2. Parâmetros de calibração para os métodos analíticos propostos para quantificar olanzapina utilizado isopropanol como solvente

\begin{tabular}{|c|c|c|c|c|c|c|}
\hline Método & $\lambda(\mathrm{nm})$ & Equação & $\mathrm{r}$ & $\operatorname{LOD}\left(\mathrm{mg} \mathrm{L}^{-1}\right)$ & $\operatorname{LOQ}\left(\mathrm{mg} \mathrm{L}^{-1}\right)$ & $\operatorname{LOL}\left(\mathrm{mg} \mathrm{L}^{-1}\right)$ \\
\hline $\mathrm{OZ}$ & 273,0 & $A=0,026+0,064 C$ & 0,9999 & 0,170 & 0,516 & 50,0 \\
\hline $\mathrm{OZ}$ & 226,5 & $A=0,043+0,075 C$ & 0,9998 & 0,205 & 0,620 & 45,0 \\
\hline $1 \mathrm{D}$ & 297,0 & $\mathrm{D} 1=-0,029-0,116 \mathrm{C}$ & $-0,9999$ & 0,106 & 0,321 & 50,0 \\
\hline $2 \mathrm{D}$ & 310,0 & $\mathrm{D} 2=0,029+0,110 \mathrm{C}$ & 0,9999 & 0,040 & 0,123 & 50,0 \\
\hline $2 \mathrm{D}$ & 275,5 & $\mathrm{D} 2=-0,036-0,140 \mathrm{C}$ & $-0,9999$ & 0,026 & 0,081 & 50,0 \\
\hline
\end{tabular}

OZ - Ordem zero, 1D - Primeira ordem e 2D Segunda ordem. LOD - Limite de detecção, LOQ - Limite de quantificação e LOL - Limite de linearidade

Tabela 3. Precisão intradia, interdia e interlaboratorial para os métodos utilizando etanol como solvente

\begin{tabular}{|c|c|c|c|c|c|c|}
\hline Método $(\lambda, \mathrm{nm})$ & $\begin{array}{c}\text { Precisão Intradia } \\
\text { Concentração }\left(\mathrm{mg} \mathrm{L}^{-1}\right)\end{array}$ & $\begin{array}{l}\text { Analista } 1 \\
\text { DPR }^{*}(\%)\end{array}$ & $\begin{array}{c}\text { Precisão Interdia } \\
\text { Concentração }\left(\mathrm{mg} \mathrm{L}^{-1}\right)\end{array}$ & $\begin{array}{l}\text { Analista } 2 \\
\text { DPR }^{*}(\%)\end{array}$ & $\begin{array}{c}\text { Precisão Interlab. } \\
\text { Concentração }\left(\mathrm{mg} \mathrm{L}^{-1}\right)\end{array}$ & $\begin{array}{l}\text { Analista } 3 \\
\operatorname{DPR}^{*}(\%)\end{array}$ \\
\hline \multirow{3}{*}{$\begin{array}{l}\text { OZ } \\
(272,5)\end{array}$} & 5,0 & 0,6 & 5,0 & 0,9 & 5,0 & 4,8 \\
\hline & 10,0 & 0,9 & 10,0 & 0,8 & 10,0 & 3,8 \\
\hline & 15,0 & 0,7 & 15,0 & 1,1 & 15,0 & 2,2 \\
\hline \multirow{3}{*}{$\begin{array}{l}\text { OZ } \\
(226,5)\end{array}$} & 5,0 & 0,9 & 5,0 & 1,3 & 5,0 & 4,4 \\
\hline & 10,0 & 0,7 & 10,0 & 0,8 & 10,0 & 4,0 \\
\hline & 15,0 & 1,1 & 15,0 & 1,0 & 15,0 & 2,6 \\
\hline \multirow{3}{*}{$\begin{array}{l}1 \mathrm{D} \\
(296,5)\end{array}$} & 5,0 & 0,4 & 5,0 & 0,6 & 5,0 & \\
\hline & 10,0 & 0,1 & 10,0 & 0,1 & 10,0 & \\
\hline & 15,0 & 0,7 & 15,0 & 0,8 & 15,0 & \\
\hline \multirow{3}{*}{$\begin{array}{l}2 \mathrm{D} \\
(310,0)\end{array}$} & 5,0 & 0,6 & 5,0 & 0,8 & 5,0 & \\
\hline & 10,0 & 0,5 & 10,0 & 0,6 & 10,0 & \\
\hline & 15,0 & 0,9 & 15,0 & 0,7 & 15,0 & \\
\hline \multirow{3}{*}{$\begin{array}{l}2 \mathrm{D} \\
(275,0)\end{array}$} & 5,0 & 0,7 & 5,0 & 1,0 & 5,0 & \\
\hline & 10,0 & 1,0 & 10,0 & 0,7 & 10,0 & \\
\hline & 15,0 & 0,8 & 15,0 & 1,0 & 15,0 & \\
\hline
\end{tabular}

DPR - Desvio padrão relativo. OZ - Ordem zero, 1D - Primeira ordem e 2D Segunda ordem

detecção $\left(0,026 \mathrm{mg} \mathrm{L}^{-1}\right)$ e de quantificação $\left(0,081 \mathrm{mg} \mathrm{L}^{-1}\right)$ foram obtidos para o método $2 \mathrm{D}$ em $275,5 \mathrm{~nm}$. Estes valores são menores que os dos métodos espectrofotométricos ${ }^{10,11}$ que utilizam reagentes tóxicos e reações $\left(>0,5 \mu \mathrm{g} \mathrm{mL}^{-1}\right)$, elétroforéticos ${ }^{10}\left(>0,3 \mu \mathrm{g} \mathrm{mL}^{-1}\right)$ e voltamétricos ${ }^{10}(>$ $\left.3,0 \mu \mathrm{g} \mathrm{mL}^{-1}\right)$, porém maiores quando comparados aos dos métodos cromatográficos ${ }^{8,9}$ (HPLC) que apresentam valores inferiores a $10 \mathrm{ng} \mathrm{mL}^{-1}$.

\section{Precisão}

O estudo de precisão foi realizado somente com as soluções do comprimido Zyprexa $^{\circledR}$, pois esta amostra é mais complexa e envolve mais etapas no processo de preparo. Os resultados para as precisões intradia, interdia e interlaboratorial encontram-se na Tabela 3.

Observa-se que, para todos os métodos usando etanol como solvente, os desvios- padrão relativos (DPR) foram menores que o máximo estabelecido pela $\operatorname{ANVISA}^{14}(5,0 \%)$. A precisão intermediária foi avaliada comparando-se os resultados obtidos entre dois analistas, em dias distintos, no mesmo laboratório. Isto foi realizado a fim de se verificar se no mesmo laboratório o método fornece os mesmos resultados. ${ }^{13} \mathrm{~A}$ comparação, através teste $F$ e teste $t$ pareado $(\mathrm{p}=0,05)$, mostrou não haver diferença significativa nos resultados obtidos pelos dois analistas.

Para avaliar o grau de reprodutibilidade dos métodos propostos, foi realizado um ensaio interlaboratorial no laboratório da Farmácia Escola da UFPI, por um analista que nunca havia trabalhado com o método e o executou em todas as etapas, desde o preparo da amostra, obtenção dos espectros, construção da curva de calibração, até os ensaios de precisão. Observa-se, na Tabela 3, que os desvios-padrão relativos foram maiores quando comparados aos dos ensaios intra e interdia, o que já era esperado devido às limitações do espectrofotômetro (Techcomp) utilizado pelo analista 3 , que não permitia ajuste das condições de varredura, como abertura de fenda, intervalo de comprimento de onda e leitura e velocidade de varredura. $\mathrm{O}$ estudo foi realizado apenas para ordem zero, pois o programa computacional do equipamento não permitia a variação do valor de $\Delta \lambda$ na derivação do espectro, o que representa uma limitação dos métodos derivativos. Outro fator que pode ter levado a desvios maiores é que o analista 3 nunca havia aplicado os métodos, diferentemente dos analistas 1 e 2 que os desenvolveram. Apesar disso, os valores de DPR ficaram abaixo do limite máximo estabelecido pela ANVISA $(5,0 \%)$. Comparando os valores encontrados neste ensaio com o teste de repetitividade (interdia) através da aplicação do teste $F$ e teste $t$ pareado $(p=0,05)$ observa-se não haver diferença significativa entre os resultados encontrados para estes dois testes podendo, portanto, ser considerados estatisticamente iguais.

A Tabela 4 mostra os resultados de precisão utilizando isopropanol como solvente. Os valores de DPR indicam que os métodos possuem uma boa precisão (DPR $<5,0 \%$ ) nos três níveis de concentrações estudados. Verificou-se, pelos resultados dos ensaios interdia, que todos os métodos são precisos com a alteração de dia e de analista e produzem resultados que não se diferenciam significativamente (Teste $t$ pareado e teste $F, \mathrm{p}=0,05$ ). Na avaliação da 
Tabela 4. Precisão intradia, interdia e interlaboratorial para os métodos utilizando isopropanol como solvente

\begin{tabular}{|c|c|c|c|c|c|c|}
\hline Método $(\lambda, \mathrm{nm})$ & $\begin{array}{c}\text { Precisão Intradia } \\
\text { Concentração }\left(\mathrm{mg} \mathrm{L}^{-1}\right)\end{array}$ & $\begin{array}{l}\text { Analista } 1 \\
\operatorname{DPR}^{*}(\%)\end{array}$ & $\begin{array}{c}\text { Precisão Interdia } \\
\text { Concentração }\left(\mathrm{mg} \mathrm{L}^{-1}\right)\end{array}$ & $\begin{array}{l}\text { Analista } 2 \\
\operatorname{DPR}^{*}(\%)\end{array}$ & $\begin{array}{c}\text { Precisão Interlab. } \\
\text { Concentração }\left(\mathrm{mg} \mathrm{L}^{-1}\right)\end{array}$ & $\begin{array}{l}\text { Analista } 3 \\
\operatorname{DPR}^{*}(\%)\end{array}$ \\
\hline \multirow{3}{*}{$\begin{array}{l}\mathrm{OZ} \\
(273,0)\end{array}$} & 5,0 & 0,7 & 5,0 & 1,8 & 5,0 & 2,4 \\
\hline & 10,0 & 1,2 & 10,0 & 1,1 & 10,0 & 2,1 \\
\hline & 15,0 & 1,6 & 15,0 & 1,3 & 15,0 & 2,6 \\
\hline \multirow{3}{*}{$\begin{array}{l}\mathrm{OZ} \\
(226,5)\end{array}$} & 5,0 & 1,0 & 5,0 & 1,5 & 5,0 & 45,7 \\
\hline & 10,0 & 1,1 & 10,0 & 2,0 & 10,0 & 23,9 \\
\hline & 15,0 & 1,6 & 15,0 & 1,8 & 15,0 & 21,9 \\
\hline \multirow{3}{*}{$\begin{array}{l}\text { 1D } \\
(297,0)\end{array}$} & 5,0 & 0,9 & 5,0 & 1,0 & 5,0 & \\
\hline & 10,0 & 1,4 & 10,0 & 1,4 & 10,0 & \\
\hline & 15,0 & 1,1 & 15,0 & 2,0 & 15,0 & \\
\hline \multirow{3}{*}{$\begin{array}{l}2 \mathrm{D} \\
(310,0)\end{array}$} & 5,0 & 1,3 & 5,0 & 1,1 & 5,0 & \\
\hline & 10,0 & 0,9 & 10,0 & 2,2 & 10,0 & \\
\hline & 15,0 & 1,6 & 15,0 & 1,6 & 15,0 & \\
\hline \multirow{3}{*}{$\begin{array}{l}2 \mathrm{D} \\
(275,5)\end{array}$} & 5,0 & 1,6 & 5,0 & 1,9 & 5,0 & \\
\hline & 10,0 & 1,9 & 10,0 & 1,4 & 10,0 & \\
\hline & 15,0 & 1,2 & 15,0 & 2,1 & 15,0 & \\
\hline
\end{tabular}

OZ - Ordem zero, 1D - Primeira ordem e 2D Segunda ordem. DPR - Desvio padrão relativo

precisão interlaboratorial, somente a banda em 273,0 nm apresentou resultados satisfatórios (com DPR $<5,0 \%$ ) e estatisticamente iguais aos do ensaio inter-dia (Teste $t$ pareado e teste $F, \mathrm{p}=0,05$ ). Os desvios obtidos para a banda a $226,5 \mathrm{~nm}$ foram bastante elevados, dessa forma esse comprimento de onda não é adequado para análise usando isopropanol como solvente. Este fato pode ser atribuído às limitações do equipamento utilizado nesse ensaio, visto que com o uso do espectrofotômetro Hitachi as precisões foram satisfatórias em ambos os comprimentos de onda.

Comparando-se o valor do desvio padrão relativo, verifica-se que os métodos utilizando etanol como solvente são mais precisos, porém todos os métodos propostos são similares em termos de precisão aos métodos espectrofotométricos que utilizam reações com a olanzapina ${ }^{11}$ (precisão intradia DPR $=0,62 \%$ e precisão interdia $\mathrm{DPR}=1,57 \%$ ) descritos na literatura e mais precisos que os métodos por difração de raio- $\mathrm{X}^{22}$ (precisão intradia $\mathrm{DPR}=2,1 \%$ e precisão interdia DPR $=7,8 \%$ ).

\section{Exatidão}

Nos estudos das amostras comerciais observou-se que o preparo do comprimido Zyprexa ${ }^{\circledR}$ envolve várias etapas. O comprimido possui um revestimento difícil de ser triturado, levando à grande heterogeneidade da amostra após pulverização, afetando diretamente a reprodutibilidade das réplicas amostrais. Outro problema é que, ao serem dissolvidas em ambos os solventes, formavam suspensões com uma forte turbidez e os primeiros ensaios de recuperação mostraram valores que variaram de 110 a 114\%, em função do espalhamento da radiação causado pelas partículas suspensas. O valor de DPR referente às replicatas, em cada nível, ficou entre 3,4 e 10,4\% evidenciando o baixo grau de reprodutibilidade da amostra em função de sua pouca homogeneidade. A derivação dos espectros resultou apenas em pequena diminuição das recuperações (107 e 109\%), mas com elevado valor de DPR. A fim de melhorar a homogeneidade da amostra, congelaram-se os comprimidos em nitrogênio líquido por cerca de 20 a $30 \mathrm{~min}$, o que tornou o revestimento quebradiço, possibilitando-se sua trituração e melhor homogeneização.
Vários autores realizaram separação dos excipientes antes da análise quantitativa da olanzapina. Raggi et al ${ }^{10}$ realizaram operações como extração, ajuste do pH, uso de banho ultrassônico, filtração e centrifugação. Neste trabalho, foram testadas a solubilização dos excipientes em banho ultrassônico e a filtração em papel de filtro e lã de vidro para a separação, no entanto, ambas se mostraram pouco eficientes, principalmente a filtração que foi muito lenta. Com a centrifugação houve total precipitação dos sólidos e obtenção de uma solução sobrenadante amarela transparente, por isso este foi o procedimento adotado para a remoção da interferência dos excipientes dos comprimidos de Zyprexa. As recuperações médias obtidas (Tabela 5), nos três níveis de concentração, variaram de 98 a $101 \%$ em etanol e de 99 a $103 \%$ em isopropanol. Isto mostra que, durante o processo de separação dos excipientes, não houve perda do padrão adicionado, pois as recuperações médias apresentaram valores adequados, com redução do DPR, assim as diferenças observadas nas recuperações são relacionadas apenas a erros aleatórios.

O procedimento completo de preparo da amostra pode ser simplificado para os comprimidos do NTF. Não foi necessário o congelamento da amostra e nem a centrifugação porque além desta não possuir revestimento, o que possibilitava a melhor homogeneização da amostra, os excipientes precipitam quantitativamente, podendo ser facilmente separados. As recuperações ficaram entre 99 e $103 \%$ em etanol e entre 98 e $103 \%$ em isopropanol, com DPR inferior a 5,0\%, mostrando que, com esse modo de preparo da amostra, não houve interferência dos excipientes. Esses resultados são semelhantes aos descritos na literatura para análise de olanzapina em formulações farmacêuticas. ${ }^{1,10,23}$

Os métodos propostos aparecem como novas alternativas para quantificação da olanzapina em formulações farmacêuticas na forma de comprimido. Eles mostraram-se mais acessíveis que os descritos na literatura, uma vez que envolvem equipamentos mais simples, baratos e de fácil manipulação. São também mais seguros e baratos, visto que empregam reagentes de baixa toxicidade e de baixo custo. Os métodos inovam quanto ao preparo da amostra, pois com o congelamento, sem nenhum equipamento especial, obtém-se homogeneidade adequada. Os longos processos como extração e filtração, para eliminação dos interferentes (excipientes), são substituídos por centrifugação, garantindo mais 
Tabela 5. Teste de adição e recuperação de padrão para os métodos propostos

\begin{tabular}{|c|c|c|c|c|c|c|c|c|c|}
\hline \multicolumn{5}{|l|}{ Etanol } & \multicolumn{5}{|c|}{ Isopropanol } \\
\hline $\begin{array}{l}\text { Método } \\
(\lambda, \mathrm{nm})\end{array}$ & $\begin{array}{c}\text { Adicionado } \\
\left(\mathrm{mg} \mathrm{L}^{-1}\right)\end{array}$ & $\begin{array}{c}\text { Rec. } \\
\left(\mathrm{mg} \mathrm{L}^{-1}\right)\end{array}$ & $\begin{array}{l}\text { Rec. } \\
(\%)\end{array}$ & $\begin{array}{c}\text { DPR } \\
(\%)\end{array}$ & $\begin{array}{l}\text { Método } \\
(\lambda, \mathrm{nm})\end{array}$ & $\begin{array}{l}\text { Adicionado } \\
\left(\mathrm{mg} \mathrm{L}^{-1}\right)\end{array}$ & $\begin{array}{c}\text { Rec. } \\
\left(\mathrm{mg} \mathrm{L}^{-1}\right)\end{array}$ & $\begin{array}{l}\text { Rec. } \\
(\%)\end{array}$ & $\begin{array}{c}\text { DPR } \\
(\%)\end{array}$ \\
\hline \multirow{3}{*}{$\begin{array}{l}\mathrm{OZ} \\
(272,5)\end{array}$} & 5,0 & 5,0 & 100 & 1,9 & \multirow{3}{*}{$\begin{array}{c}\mathrm{OZ} \\
(272,5)\end{array}$} & 5,0 & 4,9 & 99 & 4,0 \\
\hline & 10,0 & 10,1 & 101 & 0,6 & & 10,0 & 9,9 & 99 & 2,9 \\
\hline & 15,0 & 15,1 & 101 & 0,9 & & 15,0 & 15,1 & 101 & 1,1 \\
\hline \multirow{3}{*}{$\begin{array}{l}\mathrm{OZ} \\
(226,5)\end{array}$} & 5,0 & 5,0 & 101 & 3,4 & \multirow{3}{*}{$\begin{array}{c}\mathrm{OZ} \\
(226,5)\end{array}$} & 5,0 & 5,1 & 102 & 1,8 \\
\hline & 10,0 & 10,1 & 101 & 0,9 & & 10,0 & 9,9 & 99 & 2,9 \\
\hline & 15,0 & 15,3 & 101 & 1,0 & & 15,0 & 15,1 & 101 & 2,0 \\
\hline \multirow{3}{*}{$\begin{array}{l}1 \mathrm{D} \\
(296,5)\end{array}$} & 5,0 & 5,0 & 99 & 1,1 & \multirow{3}{*}{$\begin{array}{c}1 \mathrm{D} \\
(297,0)\end{array}$} & 5,0 & 4,9 & 99 & 2,3 \\
\hline & 10,0 & 10,0 & 100 & 0,9 & & 10,0 & 9,9 & 99 & 0,9 \\
\hline & 15,0 & 15,1 & 101 & 0,9 & & 15,0 & 14,9 & 99 & 1,2 \\
\hline \multirow{3}{*}{$\begin{array}{l}\text { 2D } \\
(310,0)\end{array}$} & 5,0 & 4,9 & 98 & 1,1 & \multirow{3}{*}{$\begin{array}{c}2 \mathrm{D} \\
(310,0)\end{array}$} & 5,0 & 5,2 & 103 & 2,8 \\
\hline & 10,0 & 10,0 & 100 & 2,2 & & 10,0 & 10,2 & 102 & 1,0 \\
\hline & 15,0 & 15,0 & 100 & 0,8 & & 15,0 & 15,3 & 102 & 0,4 \\
\hline \multirow{3}{*}{$\begin{array}{l}2 \mathrm{D} \\
(275,0)\end{array}$} & 5,0 & 5,2 & 101 & 2,2 & \multirow{3}{*}{$\begin{array}{c}\mathrm{OZ} \\
(275,5)\end{array}$} & 5,0 & 5,0 & 99 & 3,4 \\
\hline & 10,0 & 10,3 & 101 & 2,7 & & 10,0 & 10,1 & 101 & 1,8 \\
\hline & 15,0 & 15,3 & 100 & 1,3 & & 15,0 & 15,2 & 101 & 1,4 \\
\hline
\end{tabular}

DPR - Desvio padrão relativo. OZ - Ordem zero, 1D - Primeira ordem e 2D Segunda ordem. Rec. Recuperações médias

rapidez e seletividade. O processo de análise é realizado diretamente a partir do sinal do espectro obtido, sem requerer nenhum tipo de reação com a olanzapina. O emprego da espectrofotometria derivativa mostrou ser uma boa alternativa para o aumento da sensibilidade do método, no entanto, todos apresentaram resultados estatisticamente equivalentes, com boa exatidão e precisão. O método de ordem zero, devido a sua simplicidade, é facilmente aplicado. O uso do etanol como solvente é mais indicado que o isoproponol, devido a sua menor toxicidade e por conferir menor custo à análise. Os métodos desenvolvidos podem ser empregados em controle de qualidade da matéria-prima e do produto final (comprimidos) de olanzapina em indústrias farmacêuticas, uma vez que foram validados atendendo às exigências do guia da ANVISA RE n ${ }^{\circ} 899 / 2003$.

\section{CONCLUSÃO}

Os métodos desenvolvidos mostraram-se simples, seguros e confiáveis. A validação comprova que são seletivos e robustos com relação à mudança de temperatura, $\mathrm{pH}$ e marca dos solventes. A aplicação da espectrofotometria derivativa resultou em aumento da sensibilidade de alguns dos métodos, no entanto todos (OZ, 1D, 2D) tiveram resultados equivalentes nos dois solventes estudados. Os métodos apresentaram-se mais exatos e precisos quando comparados a alguns descritos na literatura para a quantificação de olanzapina em formulações farmacêuticas na forma de comprimido, podendo ser utilizados para controle de qualidade da indústria farmacêutica.

\section{AGRADECIMENTOS}

Ao CNPq, pela bolsa de iniciação científica de J. I. de Moura e pelo apoio financeiro (Processo 402.669/2005-3), à CAPES, pela bolsa de mestrado de J. F. do Rêgo, à Farmácia Escola (NTF/UFPI), pelo uso de seu laboratório e à aluna de Mestrado em Química da UFPI, J. F. de Medeiros, pela execução dos ensaios de precisão interlaboratorial.

\section{REFERÊNCIAS}

1. Jasiñka, A.; Nalewajko, E.; Anal. Chim. Acta 2004, 508, 165

2. Cavalla, D.; The Newsletter for Society for Medicines Research 2000, 6, 6.
3. http://www.pslgroup.com/dg/1ea5da.htm, acessada em Dezembro 2009.

4. Frota L. H.; Cinqüenta Anos de Medicamentos Antipsicóticos em Psiquiatria, $1^{\text {a }}$ ed CD-Rom, Dilem Informática: Rio de Janeiro, 2003.

5. Oliveira I. R.; Rev. Bras. Psiquiatr. 2000, 22 (Supl. 1), 38.

6. Marangell, L. B.; Martinez, J. M.; Silver, J. M.; Yodofsky, S. C.; Psicofarmacologia, Artmed: Porto Alegre, 2004.

7. Rao, R. N.; Raju, A. N.; Narsimha, R.; Babu, G. R.; J. Sep. Sci. 2008, $31,107$.

8. Saracino, M. A.; Gandolfi, O.; Dall'Olio, R.; Albers, L.; Kenndler, E.; Raggi, M. A.; J. Chromatogr., A 2006, 1122, 21.

9. Nirogi, R. V. S.; Kandikere, V. N.; Shukla, M.; Mudigonda, K.; Maurya, S.; Boosi, R.; Yerramilli, A.; J. Pharm. Biomed. Anal. 2006, 41, 975.

10. Raggi, M. A.; Casamenti, G.; Mandrioli, R.; Izzo, G.; Kenndler, E.; J. Pharm. Biomed. Anal. 2000, 23, 973.

11. Krebs, A.; Starczwska, B.; Puzanowska-Tarasiewicz, H.; Sledz , J.; Anal. Sci. 2006, 22, 829.

12. Firdous, S.; Aman, T.; Nisa, A.; J. Chem. Soc. Pak. 2005, 27, 163.

13. Mohamed, A. A.; Monatsh. Chem. 2008, 139, 1005.

14. Agência Nacional de Vigilância Sanitária (ANVISA); Resolução RE $n^{o}$ 899, de 29/05/2003.

15. Miller, J. N.; Miller, J. C.; Estadística y Quimiometría para Química Analítica, 4 ${ }^{\mathrm{a}}$ ed, Pearson Educacíon: Madrid, 2002.

16. Ribani, M.; Botolli, C. B. G.; Collins, C. H.; Jardim, I. C. S. F.; Melo, L. F. C.; Quim. Nova 2004, 27, 771.

17. Leite, F.; Validação em Análise Química, 4ae ed., Editora Átomo: Campinas, 2002.

18. Wawrzycka-Gorczyca, I.; Borowski, P.; Osypiuk-Tomasik, J.; Mazur. L.; Koziol, A. E.; J. Mol. Struct. 2007, 830, 188.

19. Silverstein, R. M.; Bassler, G. C.; Morril, T. C.; Identificação Espectrométrica de Compostos Orgânicos, $5^{\mathrm{a}}$ ed., LTC: Rio de Janeiro, 1994.

20. Perkampus, H. H.; UV-VIS - spectroscopy and its applications, Verlag - Springer: Hildelberg, 1992.

21. Görög, S.; Ultraviolet-Visible Spectrophotometry in Pharmaceutical Analysis, Boca Raton: CRC Press, 1995.

22. Tiwari, M.; Chawla, G.; Bansal, A. K.; J. Pharm. Biomed. Anal. 2007, 43,865 .

23. Basavaiah, K.; Rangachar, A. U.; Tharpa, K.; J. Mex. Chem. Soc. 2008, 52, 120. 